\title{
NUEVA CITA DE HORVATHINIA PELOCOROIDES MONTANDON (HEMIP- TERA: BELOSTOMATIDAE) PARA LA PROVINCIA DE CORRIENTES, ARGENTINA
}

\author{
A.C. Armúa(1); A.L. Estévez(2); A.I. $\operatorname{KeHR}^{(1)}$ y M.E. BeníteZ(1)
}

RESUMEN: Se cita a Horvathinia pelocoroides para Laguna Iberá (Corrientes, Argentina) capturada con red en su ambiente natural, se describen parcialmente las condiciones climáticas y del ambiente.

ABSTRACT: Horvathia pelocoroides is recorded for Laguna Iberá (Corrientes, Argentina) captured with net in its natural media; Climatic conditions and of the habitat are partially mentioned.

Palabras claves: Horvathinia, Belostomatidae, Nepomorpha, Heteroptera, Corrientes, Argentina, Nueva cita.

Key words: Horvathinia, Belostomatidae, Nepomorpha, Heteroptera, Corrientes, Argentina, New record.

Los Belostomatidae habitan variados ambientes acuáticos, son insectos predadores, se alimentan de los fluidos internos de invertebrados y vertebrados; se distribuyen en América, Asia y Africa. En esta familia de hemípteros acuáticos se reconocen tres subfamilias: Belostomatinae, Lethocerinae and Horvathiniinae (Lauck \& Menke, 1961; Stys \& Jansson, 1988).

El comportamiento de cuidado de los huevos, por parte de los machos es una característica singular de la mencionada familia. Slater (1899) fue el primer autor en descubrir que los machos de Belostoma portan los huevos en el dorso; luego de ese reporte Torre-Bueno (1906) y Harvey (1907) registraron y describieron el patrón de comportamiento de apareamiento y oviposición de especies de Belostoma. Rankin (1935) reconoció que las especies del género Lethocerus cuidan los huevos depositados sobre sustratos por encima de la superficie del agua.

Posteriormente a estos descubrimientos varios investigadores estudiaron e interpretaron este comportamiento (Voelker, 1968; Smith, 1976a,b; Ichikawa, 1988 Schnack et. al., 1990).

Actualmente se describen dos tipos de comportamientos de incubación de los huevos, desde la ovoposición hasta la eclosión de las larvas: "back brooding behavior" y "emergent brooding behavior".

(1) Facultad de Ciencias Exactas y Naturales y Agrimensura, UNNE, Av. Libertad 5470 (3400) Corrientes. Argentina. E-mail: acarmua@exa.unne.edu.ar

(2) División Entomología, Museo de La Plata, Universidad Nacional de La Plata, Paseo del Bosque s/n, (1900) La Plata, Argentina. E-mail: anitaestevez@argentina.com 
El comportamiento de incubación de los huevos en el dorso de los machos, se reconoce como un comportamiento reproductivo obligatorio en los Belostomatinae; las hembras ponen los huevos sobre el dorso de los machos, los machos cuidan de los huevos exponiéndolos al aire, limpiándolos, etc., revelando un patrón de comportamiento complejo ("back brooding behavior"). Los Belostomatinae incluyen especies del género Abedus Stål, 1862, Belostoma Latreille, 1807, Diplonychus Laporte, 1833, Hydrocyrius Spinola, 1850, Limnogeton Mayr, 1853 and Weberiella (Menke, 1965). Estévez \& Ribeiro $(2010)$ pueden incluir este género indiscutiblemente entre los Belostomatinae debido al descubrimiento de machos incubantes.

Los miembros de la subfamilia Lethocerinae depositan sus huevos sobre la vegetación acuática emergente (DeCarlo, 1962); los machos cuidan de los huevos manteniendo la humedad y protegiéndolos de los depredadores ("emergent brooding behavior") (Ichikawa, 1988; Smith \& Larsen, 1993; Ohba, 2002). Esta subfamilia incluye especies del genero Lethocerus Mayr, Benacus Stål and Kircaldyia.

Se desconoce el comportamiento reproductivo de la subfamilia Horvathininae. Horvathinia pelocoroides es muy infrecuente y se lo puede hallar solo en una limitada región de América del Sur. Este género monotípico es conocido desde que Montandon (1911) describió $H$. pelocoroides. Desde ese momento hasta ahora fueron descriptos como especies nuevas muy pocos ejemplares, procedentes de distintas localidades; todos fueron capturados fuera del medio acuático, atraídos por la luz artificial: Horvathinia doellojuradoi De Carlo 1930: $H$. pelleranoi De Carlo 1930, $H$. meyeri De Carlo 1938, $H$. castilloi De Carlo 1938, H. schubarti.

Sobre la base de la observación de 55 ejemplares que fueron capturados fuera del agua en 1981 y del material de tipo, Schnack y Estévez (2005) concluyeron que Horvathinia es un género monotípico.

Domizi (observ. pers.) mantuvo 35 insectos vivos, lo cual permitió que se realizaran las primeras observaciones del comportamiento de estos insectos durante marzo de 1981; formó parejas en el laboratorio y describió detalles acerca de las hembras depositando huevos semi enterrados en arena húmeda fuera del agua, sugiriendo un comportamiento diferente al de "back brooding" (Schnack et. al., 2006).

Pérez Goodwyn et al. (2006) estudiaron la estructura coriónica de los huevos de Lethocerus delpontei, Kirkaldyia deyrolei and Horvathinia pelocoroides, utilizando microscopía electrónica de barrido, concluyendo que Horvathinia pelocoroides desarrolla un comportamiento probablemente de un "emergent brooding" debido a que las características de la superficie coriónica es similar a aquellos Lethocerinae estudiados. A pesar de estas observaciones, el tipo del comportamiento reproductivo de Horvathininae sigue permaneciendo en el campo especulativo.

En Argentina este género fue encontrado en las provincias del Nordeste como Misiones (Loreto y Apóstoles), Chaco (Fontana) y Corrientes (Santo Tomé, Ituzaingó, Colonia Pellegrini y Laguna Don Blanco).

En la Laguna Don Blanco, en el departamento de San Cosme en la Provincia de Corrientes $\left(27^{\circ} 27^{\prime} 31^{\prime \prime} \mathrm{S}, 58^{\circ} 49^{\prime} 34^{\prime \prime} \mathrm{W}\right)$, en diciembre de 2004 se capturó del medio acuático, por primera vez, un Horvathinia macho y en Septiembre de 2005 se halló una hembra que fue también capturada con red (Schnack et al., 2006). 
En la presente comunicación se da a conocer un nuevo hallazgo de un Horvathinia macho de Laguna Iberá, Lobo Cuá, 28³2’27"S, 57²1'14"W (Lobo Cuá es un lugar ubicado en la ribera de Laguna Iberá, Provincia Corrientes, Argentina); la superficie de la laguna estaba cubierta de Pistia stratiotes (Araceae), Salvinia sp. (Salviniacea), Egeria densa (Hydrocharitaceae) y Eichornia crassipes (Pontederiaceae).

La extracción de las muestras se realizó el 6 de noviembre de 2010 , con una red de $45 \mathrm{~cm}$ de diámetro. El ejemplar atrapado estaba oculto entre las raíces de la vegetación; en la misma muestra fueron capturados otros Belostomatidae: B. elegans, Mayr 1863, B. micantulum Stal, 1860 y B. lopretoae Estévez y Armúa.

Las condiciones del agua en el momento del muestreo fueron: Temperatura: $27^{\circ}, 6^{\circ} \mathrm{C}$; pH: 7.2 . Condiciones climáticas: temperatura: $36.9^{\circ} \mathrm{C}$, viento leve, soleado. Hora de captura: 11:00 a.m.

El ejemplar de Horvathinia fue mantenido vivo en el laboratorio; se lo colocó en un recipiente de vidrio con agua de la laguna y Eichornia como sustrato. El insecto vivió 5 días. Solamente se pudo observar que se movía lentamente entre las raíces y que se desplazaba desde la superficie hacia el fondo del recipiente.

El macho capturado de Laguna Iberá tiene gránulos de arena adheridos al abdomen y entre las patas, al igual que los ejemplares antes capturados, en la luz o del agua. Este hecho sugiere que Horvathinia frecuentaría el fondo o las orillas de ríos o charcas. El sitio de muestreo en la laguna Don Blanco como el sitio de muestreo en la laguna Iberá estaba cubierto por la misma vegetación.

Todavía es un enigma si Horvathinia realiza alguna especie de cuidado de los huevos, similar a aquellos de Belostomatinae o Lethocerinae o si tiene un comportamiento diferente.

Con el conocimiento de los hábitats donde es posible encontrar Horvathinia, se espera acceder a material suficiente como para poder estudiar Horvathiniinae en forma exhaustiva y resolver su status taxonómico.

\section{BIBLIOGRAFÍA}

De CARı.o, J.M., 1962. Consideraciones sobre la biología de Lethocenus mazzai /l. Physis Sec. B., 23 (65): $143-151$.

Est'́́vi\%, A.L. and J.I. Ristirro, 2010. Weberiella De Carlo, 1966 (Insecta: Heteroptera: Belostomatidae) revisited: Redescription with a key to the genera of Belostomatidae and considerations on backbrooding behaviour. Zoologischer Anzeiger, 250 (2011): 46-54.

HARVEY, G.W., 1907. A ferocious water bug. Can. Entomol., 39: 17-21.

ICHIKAWA. N., 1988. Male brooding behaviour of the giant water bug Lethocerus deyrollei Vuillefroy (Hemiptera: Belostomatidae). Journal of Ethology, Vol. 6: 121-127.

LAUCK, D.R. and A.S. MENKE, 1961. The higher classification of the Belostomatidae (Hemiptera). Annals of Entomological Society of America, 54: 644-657.

Montanion, A.L., 1911. Deux genres nouveaux d'hydrocorises. Annals. Mus. Nat. Hungr., 9: 244-246. 
Онва, S., 2002. Synchronized mechanism and its meaning in the egg hatching of the water bug, Lethocerus deyrolli (Heteroptera: Belostomatidae). Japanese Joumal of Entomology, Jpn. J. Ent. (N.S.), 5 (4): 157-164.

PÉreZ-Goodwyn, P.J.; S. OHBA and J.A. SchNACK, 2006. Chorion morphology of the eggs of Lethocertus delpontei, Kirkaldyia deyrolli, and Horvathinia pelocoroides(Heteroptera: Belostomatidae). Russian Entomological Journal, 15 (2): 1-6.

RANKIN, K. and A. EsTEVEZ, 1935. Life history of Lethocerus americanus Leidy (Belostomatidae, Hemiptera). Kansas University Sciences Bulletin, 36: 479-491.

SCHNACK, J.A.; E.A. DomIZI and A.L. EstéveZ, 1990. Comportamiento reproductivo de Belostoma oxymmm (Hemiptera: Belostomatidae). Revista de la Sociedad Entomológica Argentina, 48: 121-128.

SCHNACK, J.A. and A.L Estévez, 2005. On the taxonomic status of the genus Horvathinia Montandon (Hemiptera: Belostomatidae). Zootaxa. ISSN $1175-5326$ (print edition). ISSN 1175-5334 (online edition).

SchnAck, J.A.; A.L Est'́́vt\% and A.C. ArmúA de Rtyts, 2006. Laguna Don Blanco, Argentina: first record of Horvathinia (Hemiptera:Belostomatidae) as a wetland dweller. Entomological News, Volume 117. Number 2: 197-202.

Slater, F.W., 1899. The egg carrying habit of Zaitha. Ann. Nat., 33: 931-933.

SMITH, R.L., 1976a. Male brooding behavior of the water bug Abedus herberti (Heteroptera: Belostomatidae). Annals of Entomological Society of America, 69: 740-747.

SMITH, R.L., 1976b. Brooding behavior of a male water bug Belostoma flumineum (Hemiptera: Belostomatidae). Kansas Entomological Society, 49: 333-343.

SMITH, R.L. and E. LARStN, 1993. Egg attendance and brooding by males of the giant water bug Lethocerus medius (Guerin) in the field (Heteroptera: Belostomatidae). Joumal of Insect Behavior, 6: 93-106.

ŚrYY, P. and A. JANSSON, 1988. Check-list of recent family-group and genus-group names of Nepomorpha (Heteroptera) of the world. Acta Entomologica Fennica, 50: 1-44.

TORRE-BuENo de la J.R., 1906. Life histories of North American water bugs. Canadian Entomologist, 38 : 189-197.

VoI.KFR, J., 1968. Untersuchungen zu Emahrung, Fortpflanzungsbiologie und Entwicklung von Limnogeton fieberi Mayr (Belostomatidae: Hemiptera) als Beitrag zur Kenntnis von naturlichen Feinden tropischer SuBwas-verschnecken. Entomologische Mitteilungen Staatsinst aus dem Zoologischen Museum Hamburg. 3: 1-24. 